\title{
Telomerase is an independent prognostic marker of overall survival in patients with colorectal cancer
}

\begin{abstract}
R Bertorelle ${ }^{1}$, M Briarava ${ }^{2}$, E Rampazzo ${ }^{3}$, L Biasini ${ }^{1}, \mathrm{M} \mathrm{Agostini}^{2}$, I Maretto ${ }^{4}$, S Lonardi ${ }^{5}, \mathrm{M} \mathrm{L} \mathrm{Friso}{ }^{6}, \mathrm{C} \mathrm{Mescoli}^{7}$,

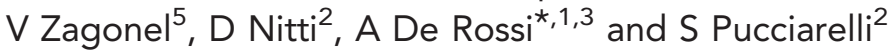

${ }^{1}$ Immunology and Molecular Oncology Unit, Istituto Oncologico Veneto, IRCCS, Via Gattamelata 64, 35128 Padova, Italy; 2 Department of Surgical Sciences, Oncology and Gastroenterology, Section of Surgery, Via Giustiniani 1, University of Padova, 35128 Padova, Italy; ${ }^{3}$ Department of Surgical Sciences, Oncology and Gastroenterology, Section of Oncology and Immunology, Via Gattamelata 64, University of Padova, 35128 Padova, Italy; ${ }^{4}$ Surgical Unit, Istituto Oncologico Veneto, IRCCS, Via Gattamelata 64, 35128 Padova, Italy; ${ }^{5}$ Medical Oncology Unit 1, Istituto Oncologico Veneto, IRCCS, Via Gattamelata 64, 35128 Padova, Italy; ${ }^{6}$ Radiotherapy and Nuclear Medicine Unit, Istituto Oncologico Veneto, IRCCS, Via Giustiniani 1, 35128 Padova, Italy and ${ }^{7}$ Department of Medical Diagnostic Sciences and Special Therapies, Pathology and Cytopathology Unit, Via Gabelli, University of Padova, 35128 Padova, Italy
\end{abstract}

Background: Colorectal cancer (CRC) is an important cause of cancer-related death. Prediction of recurrence is an important issue in the treatment of disease, particularly for stage II patients. The level of telomere-specific reverse transcriptase (hTERT), the catalytic component of the telomerase complex, increases along with CRC progression, but its prognostic value is still unclear.

Methods: One hundred and thirty-seven CRC patients were studied for hTERT expression in tumour cells by real-time PCR. hTERT level was evaluated as a prognostic factor of overall survival (OS) in all patients and of disease recurrence in a subgroup of 50 stage II patients.

Results: The median hTERT level was 93.8 copies (interquartile range 48-254). Patients with high hTERT levels (above the median) showed a significantly worse survival than those with low hTERT levels (below the median; log-rank test $P<0.0001$; hazard ratio $(H R)=3.30$ (95\% confidence interval $(C l) 1.98-5.52) ; P<0.0001)$. The negative prognostic value of high $h T E R T$ level is independent of the pathological stage and microsatellite instability $(\mathrm{HR}=2.09(95 \% \mathrm{Cl} 1.20-3.64), P=0.009)$. Moreover, in stage II $\mathrm{CRC}$, high hTERT levels identified patients with a higher risk of disease recurrence $(H R=3.06(95 \% \mathrm{Cl} 1.03-9.04), P=0.043)$ and death $(\mathrm{HR}=3.24(95 \% \mathrm{Cl} 1.37-7.71), P=0.008)$.

Conclusion: hTERT level is an independent prognostic marker of OS in CRC patients. In addition, assessment of hTERT level could improve stratification of stage II CRC patients for the risk of disease recurrence.

Colorectal cancer (CRC) is the third most commonly diagnosed cancer in males and the second in females, with over 1.2 million new cases and 608700 deaths estimated to have occurred worldwide in 2008 (Jemal et al, 2011). Despite improved treatments, increased awareness and early detection which have all contributed to prolonged survival, CRC is still an important cause of cancer-related deaths.

CRC is a heterogeneous complex of diseases with different molecular pathways and biological characteristics, arising through a multistep process of which several genetic and epigenetic events 
have been characterised (Markowitz and Bertagnolli 2009). Although surgical resection and adjuvant chemotherapy are effective curative treatments, the risk of recurrence cannot be foreseen, even in patients with tumours at the same stage. Although 5-fluorouracil-based adjuvant chemotherapy is the standard of care for patients with stage III CRCs, the role of adjuvant therapy for stage II CRCs is still controversial (Andrè et al, 2009). It is therefore critical to identify the subgroup of patients with stage II tumours at high risk of recurrence; however, this is still difficult owing to the molecular characteristics of these tumours as well as their intrinsic heterogeneity (Engstrom et al, 2009; O'Connor et al, 2011).

Many efforts have been made to identify molecular markers that predict the outcome of CRC patients, and several genetic and epigenetic alterations involved in the development of CRC have been proposed as prognostic markers of disease progression. Genetic instability has a critical role in the carcinogenetic process. Most CRCs have chromosomal instability (Pino and Chung, 2010), while other CRCs have a high-grade microsatellite instability (MSI) phenotype, generated by a deficient DNA mismatch repair pathway, frequently associated with the GpG island methylator phenotype (Boland and Goel, 2010). KRAS and TP53 gene mutations, involved in chromosome instability, and the MSI phenotype have been extensively studied for their potential prognostic and predictive roles. Although a predictive value of KRAS mutation status for response to EGFR-targeted therapy has been defined (Van Cutsem et al, 2009; Cunningham et al, 2010), its prognostic value is still unclear (Menin et al, 2006; Kim et al, 2007; Cunningham et al, 2010).

Telomere/telomerase interplay is an important mechanism involved in the maintenance of genetic chromosome stability and its dysfunction has emerged as having a causative role in carcinogenesis. Telomeres provide genomic stability by protecting chromosome ends. When the shortening of telomeres, which occurs at each cell division cycle because of end-replication problems of DNA polymerase, reaches a critical length, cells cease to proliferate and undergo senescence (Blackburn et al, 2006). Further erosion of telomeres may impair their function, resulting in genetic instability (Calado and Young, 2009; Artandi and DePinho 2010). Several studies demonstrated that telomeres were shorter in CRCs than in adjacent mucosa, suggesting that telomere shortening is a key initial event in colorectal carcinogenesis (Hastie et al, 1990; Takagi et al, 1999; Gertler et al, 2004; Garcia-Aranda et al, 2006; Rampazzo et al, 2010). Nevertheless, the maintenance of telomere length by telomerase is critical to preserving the replicative potential of cancer cells. Telomerase is a ribonucleoprotein complex containing a catalytic protein with telomerespecific reverse transcriptase (hTERT), which synthesizes telomeric sequences de novo utilising an internal RNA template (hTR). hTERT is the rate-limiting component of the telomerase complex (Nakamura et al, 1997) and its expression, usually absent from normal somatic cells, is essential for unlimited cell growth (Kelland, 2007). Notably, recent studies have suggested that, besides maintenance of telomere length, hTERT is involved in several other cellular functions. Expression of hTERT increases replicative potential (Rufer et al, 2001; Terrin et al, 2007), promotes cell growth in adverse conditions and may act as an anti-apoptotic agent (Del Bufalo et al, 2005; Rahaman et al, 2005).

Previous studies have shown that hTERT expression and/or telomerase activity is higher in CRCs than in adjacent noncancerous mucosa (Gertler et al, 2002; Nowak et al, 2003; GarciaAranda et al, 2006; Terrin et al, 2008) and increases along with cancer progression (Maláska et al, 2004; Terrin et al, 2008; Rampazzo et al, 2010). However, the prognostic value of telomerase is controversial. Different results may be partly owing to the assessment of different end-points, disease-free survival (DFS) or overall survival (OS) and/or variations in the methodology employed to measure this marker, that is, telomerase activity or hTERT genetic expression (Tatsumoto et al, 2000; Kawanishi-Tabata et al, 2002; Gertler et al, 2004; Garcia-Aranda et al, 2006; Sanz-Casla et al, 2005; Vidaurreta et al, 2007; Safont et al, 2011). Of note, the only study, to the best of our knowledge, that addressed the prognostic role of telomerase in patients with stage II CRCs found that positivity for telomerase activity was associated with a better clinical outcome (Kawanishi-Tabata et al, 2002). In previous studies we developed a real-time PCR assay to quantitate hTERT mRNA, and have found that levels of hTERT expression correlated with telomerase activity and increased with tumour stage and histological grade (Terrin et al, 2008; Rampazzo et al, 2010).

The aim of this study was to elucidate the role of hTERT expression, quantified in tumour tissues by real-time PCR, as a prognostic marker of survival in CRC patients. In addition, we focused our study on verifying whether hTERT levels may be a prognostic marker of disease recurrence in stage II patients.

\section{MATERIALS AND METHODS}

Patients. The study population consisted of 137 patients with a diagnosis of CRC who underwent surgery at a single Institution from 1995 to 2005. The surgical specimens, assessed in a standardised way, were reviewed by one pathologist (CM), following the American Joint Committee on Cancer TNM stage system, 2010. The initial inclusion criteria of patients were the availability of tumour tissues in which the neoplastic component was at least $80 \%$ of cells, the availability of adjacent non-cancerous mucosa and an adequate clinical follow-up (116 patients). Because of interest in defining the prognostic value of hTERT levels in stage II CRC patients, an additional group of 21 patients with stage II CRCs, for whom adequate tumour samples and clinical follow-up were available, was included in the study. Although the study was retrospective, the data were prospectively recorded in an institutional database. At surgery, each patient signed the informed consent approving that biological samples could be stored at our biological bank and used for research purposes.

The MSI status, performed by analysing five microsatellites of the Bethesda recommended panel (BAT-25, BAT-26, D2S123, D5S346, and D17S250) (Pucciarelli et al, 2003), was available for all tumours (Table 1).

Quantification of hTERT transcripts. RNA samples were extracted from cryostat sections of primary tumours using Trizol reagent (Invitrogen, Life Technologies, Carlsbad, CA, USA) and were reverse transcribed into cDNA using the SuperScript TM III RNase reverse transcriptase assay (Invitrogen) (Terrin et al, 2008). The expression of hTERT transcripts was quantified by real-time PCR using an ABI prism 7900 HT Sequence Detection System (Applied Biosystems, Life Technologies). Absolute quantification was carried out using five-fold dilutions of hTERT amplicon as a reference curve for hTERT copies, and five-fold dilutions of housekeeping hypoxanthine-guanine phosphoribosyl transferase 1 (HPRT1) amplicon for HPRT1 copies. Values of hTERT were then normalised for $10^{3}$ copies of HPRT1, as previously reported (Terrin et al, 2008).

Statistical analyses. OS was considered the end-point of the study and was defined as the interval between the date of surgery and the date of death or the last follow-up. DFS was defined as the interval between the date of surgery and the date of disease recurrence. Univariate and multivariate survival analysis was performed by means of the Cox regression model. The proportional hazard assumption was tested using the Grambsch-Therneau method (proportional hazard-test), which is based on Schoenfeld residuals (Therneau and Grambsch, 2000). The Breslow method for handling ties was used while running the Cox model. For univariate analysis, the following prognostic factors were 


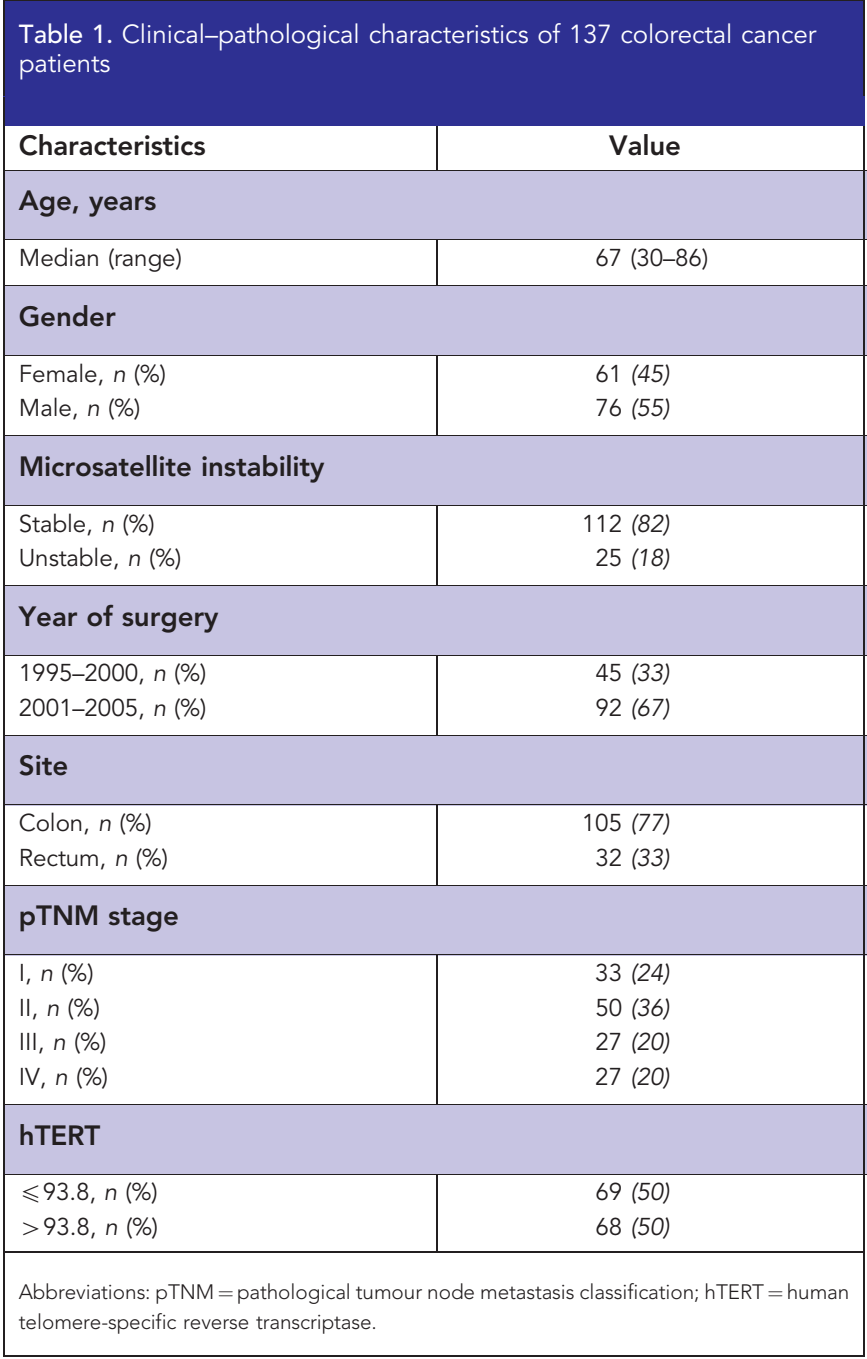

considered: age (years), site of tumours (colon vs rectum), MSI vs microsatellite stability (MSS), pTNM stage (I, II, III, IV) and hTERT levels. hTERT levels were categorised as high (above median value) and low (below median value). The fractional polynomial method was employed to study the functional form of continuous covariates and to relax the linearity assumption of the Cox regression model. The selection of the covariates that significantly and independently contribute to the prognostic model was performed using the stepwise mode. As a measure of overall prognostic accuracy of the final model we calculated the concordance index, which estimates the probability of concordance between predicted and observed events and ranges between 0.5 (no prognostic accuracy) and 1 (perfect prognostic accuracy) (Harrell et al, 1996). OS and DFS empirical curves were estimated with the Kaplan-Meier method and the survival functions were compared using the log-rank test. The median follow-up was calculated according to the reverse Kaplan-Meier method. All tests are twosided, the $\alpha$-level of significance being set to $5 \%$. All statistical analyses were performed using Stata 11 SE (StataCorp, College Station, TX, USA).

\section{RESULTS}

Characteristics of patients and relationship with hTERT expression. Clinical-pathological characteristics of the 137 CRC patients included in the study are reported in Table 1. Seventy-six patients were males and 61 were females; the median age was 67 years (range, $30-86$ years). Tumours were located in the colon in 105 cases $(77 \%)$ and in the rectum in 32 cases (23\%). According to the American Joint Committee on Cancer staging, 33 tumours were classified as stage I, 50 as stage II, 27 as stage III, and 27 as stage IV. Of the 122 patients for whom data were available, 66 received adjuvant therapy. One hundred and twelve CRCs (82\%) had stable microsatellites, whereas 25 CRCs (18\%) had MSI. Quantification of hTERT transcripts was obtained for all 137 CRC samples. The median hTERT level was 93.8 (interquartile range 48.0-254.4) copies. In accordance with previous studies (Terrin et al, 2008; Rampazzo et al, 2010), hTERT levels increased with severity of disease. Indeed, median hTERT levels were 52.4 (32.4$121.1), 85.4$ (52.8-140.0), $114.52(65.6-268.1)$ and 469.2 (210.62099.8) copies in tumours stage I, II, III, and IV, respectively (overall, $P<0.0001$ ). Furthermore, in agreement with a previous study (Rampazzo et al, 2010), no correlation was found between hTERT levels and MSI or tumour location. Median hTERT levels were $92.3(62.8-140.0)$ and $94.3(44.3-275.0)$ copies in MSI and MSS CRC, respectively $(P=0.68)$, and $106.1(54.3-275.1)$ and 80.3 $(36.2-140.1)$ copies in colon and rectal CRC, respectively $(P=0.144)$.

Relationship of hTERT level with OS of patients. With a median follow-up of 70 months (interquartile range 57-122), the 5-year survival rate was $55.4 \%$ (95\% confidence interval (CI) 46-64). With univariate analysis, TNM stage and hTERT levels were significantly associated with survival (Table 2). The survival curves according to TNM stage are shown in Figure 1A. With regard to hTERT, the study of its functional form suggested that dichotomization by the median value (93.8 copies) would yield a better fit of the survival model; considering this cutoff, patients with high hTERT levels (above the median) showed a significantly worse OS (median 37 months) than those with lower hTERT levels (below the median; (median OS not reached; log-rank test $P<0.0001$ ) (Figure $1 \mathrm{~B}$ ). This difference corresponded to a hazard ratio $(\mathrm{HR})=3.30(95 \%$ CI 1.98-5.52), $P<0.0001$.

With multivariate analysis, age, TNM stage, and hTERT were retained in the final regression model (Table 2). The prognostic value of hTERT was maintained even after adjustment for the other covariates (age, site, MSI, pTNM stage), supporting the independent role of this biomarker in predicting the OS of these patients. In particular, the risk of death for patients with high hTERT levels was approximately double that of patients with low levels $(\mathrm{HR}=2.09$ (95\% CI 1.20-3.64), $P=0.009)$. The overall prognostic accuracy of the final model was remarkable, as suggested by the high concordance index value $(0.78)$.

Considering the subgroup of 122 patients for whom the chemotherapy data were available, multivariate analysis showed that hTERT level, but not chemotherapy, was significantly associated with $\mathrm{OS}(\mathrm{HR}=2.40(95 \% 1.14-3.76), P=0.017)$. The distribution of patients who received chemotherapy did not significantly differ between patients with high or low hTERT levels (Fisher's exact test $P=0.102$ ).

Relationship of hTERT level with disease-free and OS of stage II patients. In order to evaluate the role of hTERT as a prognostic factor in patients with stage II CRC, we tested the hypothesis that hTERT might identify patients at high risk in this subgroup. As shown in Figure 2A, stage II patients with high hTERT levels showed a significantly worse median OS (55 months) than those with low hTERT levels (median OS not reached; log-rank test $P=0.0048 ; \mathrm{HR}=3.24$ (95\% CI 1.37-7.71), $P=0.008$ ). The median OS of patients with stage II disease and high hTERT levels was very similar to that of patients with stage III disease (55 and 59 months, respectively). Furthermore, as shown in Figure 2B, high hTERT levels might identify patients with higher risk of disease recurrence; 
Table 2. Univariate and multivariate survival analysis (Cox regression model).

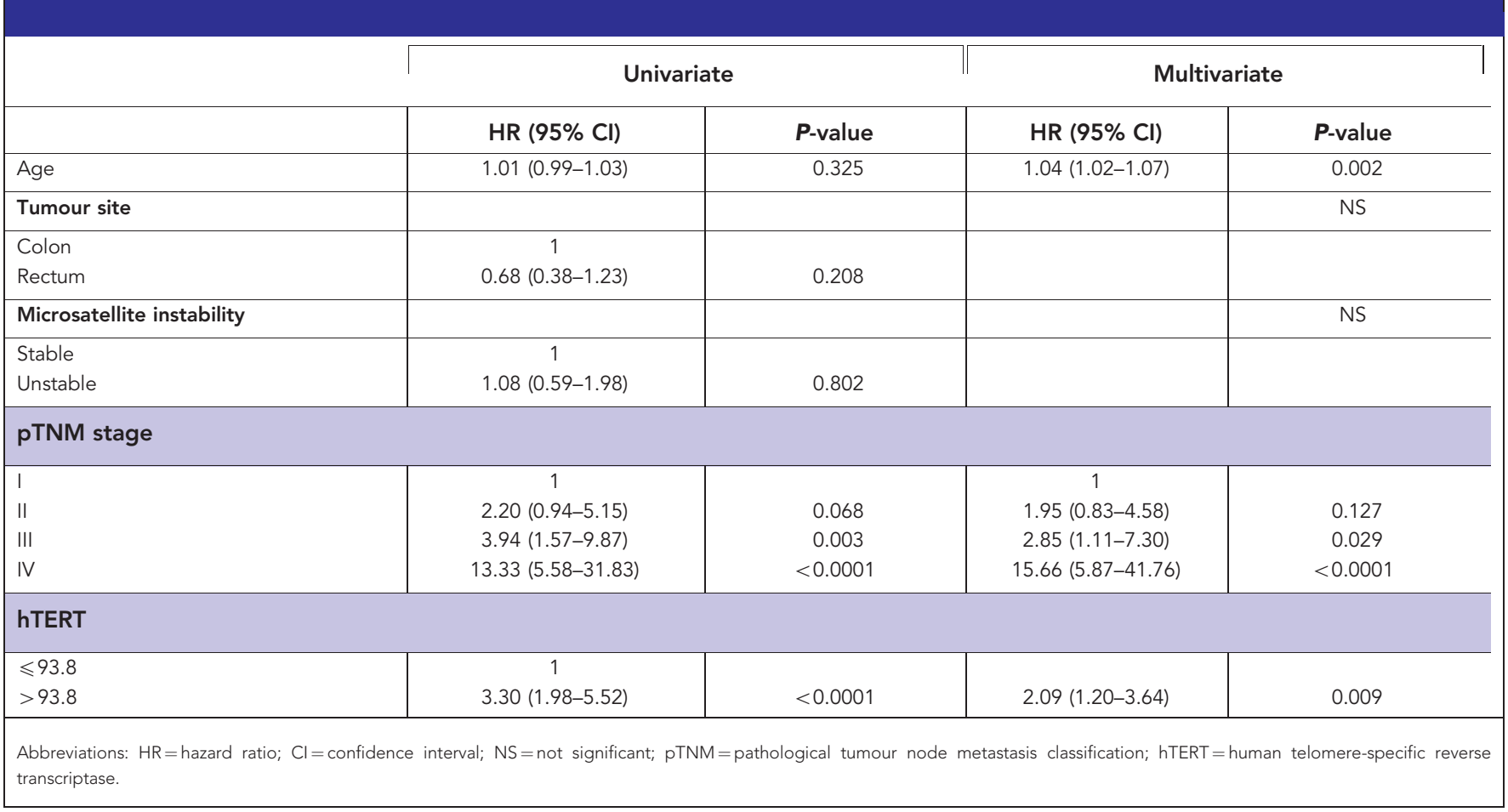

A

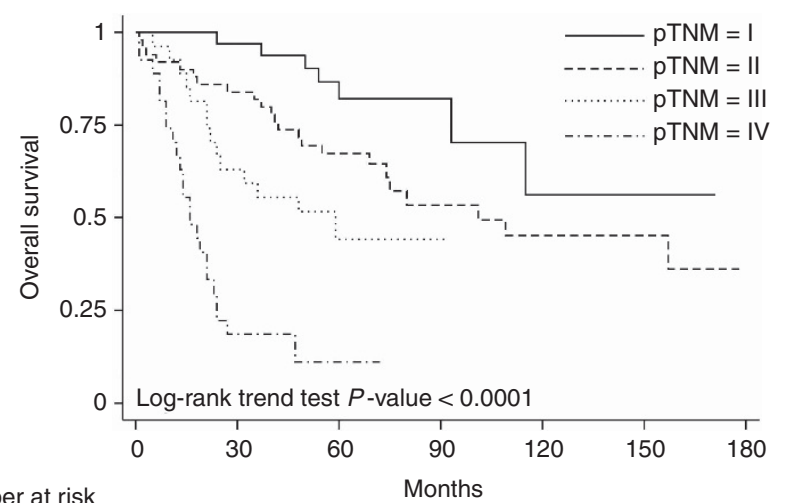

Number at risk

$\begin{array}{llllclll}\text { pTNM }=\text { I } & 33 & 32 & 19 & 7 & 4 & 4 & 0 \\ \text { pTNM }=\text { II } & 50 & 41 & 24 & 13 & 9 & 5 & 0 \\ \text { pTNM }=\text { III } & 27 & 17 & 6 & 1 & 0 & 0 & 0 \\ \text { pTNM }=\text { IV } & 27 & 5 & 2 & 0 & 0 & 0 & 0\end{array}$

B

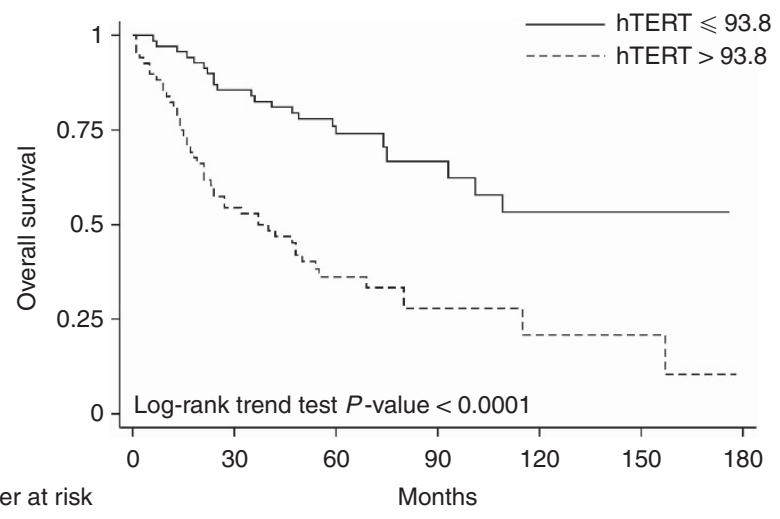

$\begin{array}{llllclll}\text { hTERT } \leqslant 93.8 & 69 & 58 & 37 & 16 & 10 & 7 & 0\end{array}$

$\begin{array}{llllllll}\text { hTERT }>93.8 & 68 & 37 & 14 & 5 & 3 & 2 & 0\end{array}$

Figure 1. (A) Kaplan-Meier OS curves of patients according to the pTNM stages. (B) Kaplan-Meier OS curves of patients according to high (above median value) or low (below median value) hTERT levels.
A

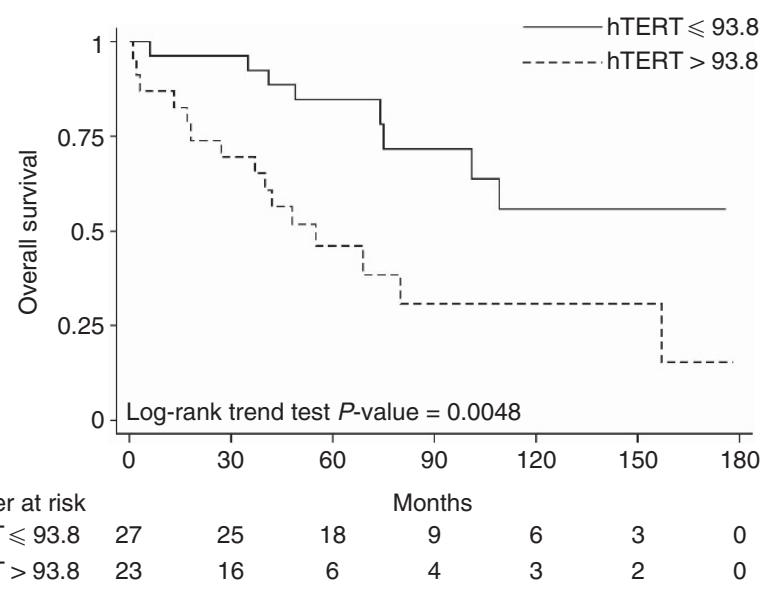

B

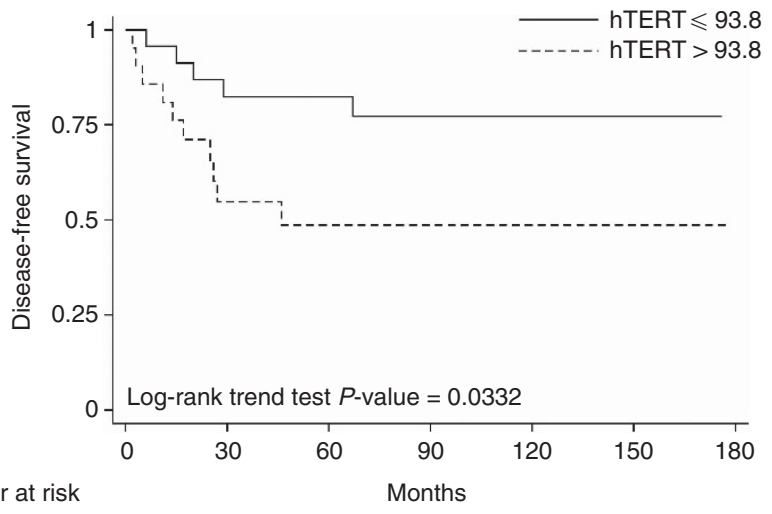

$\begin{array}{llllllll}\text { hTERT } \leqslant 93.8 & 23 & 18 & 16 & 8 & 6 & 3 & 0\end{array}$ $\begin{array}{llllllll}\text { hTERT }>93.8 & 21 & 10 & 5 & 4 & 3 & 2 & 0\end{array}$

Figure 2. (A) Kaplan-Meier OS curves of stage II patients according to high or low hTERT levels. (B) Kaplan-Meier DFS curves of stage II patients according to high or low hTERT level. 
indeed, patients with high hTERT levels showed a significantly worse median DFS (46 months) than those with low hTERT levels (median DFS not reached; log-rank test $P=0.03$; HR $=3.06(95 \%$ CI 1.03-9.04), $P=0.043$ ).

Relationship of MSI with disease-free and OS of patients. Of note, MSI status did not correlate with OS in our cohort of patients (MSI vs MSS): HR $=1.08$ (95\% 0.59-1.98), $P=0.802$ ). It has been suggested that MSI defines a subset of patients with stage II tumours with a good prognosis who do not require adjuvant 5-fluorouracil-based chemotherapy (Sargent et al, 2010). In the stage II patients we studied, 11 had tumours with MSI and 39 with MSS, 15 of whom (4 MSI and 11 MSS) received adjuvant chemotherapy. In this subset of stage II patients neither MSI nor chemotherapy had any significant impact on prognosis in terms of OS (log-rank test $P=0.231$ and $P=0.126$, respectively) and DFS (log-rank test $P=0.938$ and $P=0.502$, respectively).

\section{DISCUSSION}

Pathological tumour staging remains a key determinant of CRC prognosis and treatment. Although radical resection is the primary treatment for patients with loco-regional CRC, adjuvant chemotherapy provides additional survival benefits for patients with stage III tumours. The same benefit has not been conclusively demonstrated in patients with stage II tumours (O'Connor et al, (2011). The controversial results obtained from different studies (Ribic et al, 2003; Benson et al, 2004; Gill et al, 2004; Quasar Collaborative Group, 2007; Sargent et al, 2010) may reflect the molecular heterogeneity of CRCs and highlight the need for strong prognostic markers able to stratify patients.

In the present study, we found that a high level of hTERT is a prognostic marker of shorter OS and its negative prognostic value is independent of pathological stage. In addition, in stage II patients, a high hTERT level identified patients at higher risk of disease recurrence and death.

Several studies have addressed the prognostic value of telomerase expression in CRC, but results are quite controversial. This might be due to the different end-points assessed in these studies, DFS or OS, and the methodology employed to measure the level of hTERT expression or telomerase activity. Most of the studies utilised a semi-quantitative Telomere Repeat Amplification Protocol assay to evaluate telomerase activity (Tatsumoto et al, 2000; Kawanishi et al, 2002; Gertler et al, 2004; Garcia-Aranda et al, 2006; Sanz-Casla et al, 2005; Vidaurreta et al, 2007). Only a few studies, using different reference curves and housekeeping genes, employed real-time PCR to evaluate hTERT mRNA values (Gertler et al, 2002; Saleh and Lam 2008; Safont et al, 2011). Furthermore, only one study addressed the prognostic role of telomerase activity in patients with stage II CRCs. Positivity for telomerase activity, estimated by the Telomere Repeat Amplification Protocol assay, was associated with a better prognosis and patients with telomerasepositive CRCs had longer DFS than patients with telomerasenegative tumours (Kawanishi-Tabata et al, 2002).

In the present study, the values of hTERT were normalised for expression of the HPRT1 gene, which did not vary according to tumour stage (de Kok et al, 2005), thus allowing a more reliable estimate of hTERT levels in CRCs. Notably, hTERT levels estimated by this approach have been found to be related to telomerase activity in CRCs (Terrin et al, 2008).

Of interest, in our study, we found that hTERT levels significantly stratified stage II patients, regardless of their MSI status. MSI has been reported to be associated with improved DFS compared with MSS; however, no difference in OS was observed between MSI and MSS tumours, and MSI was not predictive of chemotherapy benefit (Kim et al, 2007). Moreover, it has been recently reported that defective DNA mismatch repair, measured by MSI or by loss of MLH1 and MSH2 proteins, is a prognostic marker of DFS; however, its prognostic effect was not maintained in multivariate models (Sargent et al, 2010). In agreement with these studies, our findings do not support the prognostic value of MSI. Indeed, OS did not significantly differ in patients with MSI and MSS tumours. It is of note that in a previous study we found that MSI tumours had shorter telomeres than MSS CRCs, but hTERT expression did not differ (Rampazzo et al, 2010). The present study, in agreement with these findings, did not find a relationship between MSI status and hTERT levels. Notably, the TP53 gene is known to be frequently altered in MSS CRCs. As the p53 is a well-known negative regulator of the hTERT promoter (reviewed in Dolcetti and De Rossi, 2012), mutated p53 may result in an earlier activation of hTERT, with an earlier stabilisation of telomeres, rather than higher levels of hTERT.

Previous studies, indicating that telomerase activity and/or hTERT expression increased along with tumour progression (Maláska et al, 2004; Terrin et al, 2008; Rampazzo et al, 2010; Kojima et al, 2011), have suggested that higher levels of hTERT may compensate for the greater shortening of telomeres due to higher proliferative activity. However, several studies, including ours, did not find a significant relationship between telomere length and telomerase levels and/or activity (Frías and Morán 2009; Rampazzo et al, 2010). Several studies suggested that hTERT might increase the malignant potential of tumours beyond just preservation of telomere length. hTERT might act as a growthpromoting and anti-apoptotic factor, independent of its telomereelongating activity (Cao et al, 2002; Del Bufalo et al, 2005; Rahman et al, 2005; Massard et al, 2006; Folini et al, 2007; Jin et al, 2010). Our findings that hTERT levels have a prognostic value, regardless of chemotherapy, support the previous in vitro observation that hTERT expression inhibited p53-dependent apoptosis in response to 5-fluorouracil in HCT116 colon carcinoma cells (Rahman et al, 2005). Specific studies are required to investigate the predictive value of hTERT levels in response to specific chemotherapies.

In conclusion, our study strongly indicates that hTERT level is an independent prognostic indicator of survival in patients with CRC. In addition, measurement of hTERT could improve the stratification of stage II patients by the risk of disease recurrence. These effects are likely associated with several functions of hTERT, in addition to its ability to maintain telomere length. Understanding the functions of hTERT is important to deepening the knowledge of CRC pathogenesis and, ultimately, to designing new therapeutic strategies, including hTERT inhibitors.

\section{ACKNOWLEDGEMENTS}

This work was supported by the Associazione Italiana Ricerca sul Cancro (AIRC). RE is a fellow recipient of AIRC. We thank Lisa Smith for editorial assistance and Pierantonio Gallo for the artwork.

\section{REFERENCES}

American Joint Committee on Cancer (2010) Colon and rectum. In: AJCC Cancer Staging Manual. 7th edn, (Springer: New York 143-164.

Andrè T, Boni C, Navarro M, Tabernero J, Hickish T, Topham C, Bonetti A, Clingan P, Bridgewater J, Rivera F, de Gramont A (2009) Improved overall survival with oxaliplatin, fluorouracil, and leucovorin as adjuvant treatment in stage II or III colon cancer in the MOSAIC trial. J Clin Oncol 27(19): 3109-3116.

Artandi SE, DePinho RA (2010) Telomeres and telomerase in cancer. Carcinogenesis 31(1): 9-18. 
Benson 3rd AB, Schrag D, Somerfield MR, Cohen AM, Figueredo AT, Flynn PJ, Krzyzanowska MK, Maroun J, McAllister P, Van Cutsem E, Brouwers M, Charette M, Haller DG (2004) American Society of Clinical Oncology recommendations on adjuvant chemotherapy for stage II colon cancer. J Clin Oncol 22(16): 3408-3419.

Blackburn EH, Greider CW, Szostak JW (2006) Telomeres and telomerase: the path from maize. Tetrahymena and yeast to human cancer and aging. Nat Med 12(10): 1133-1138.

Boland CR, Goel A (2010) Microsatellite instability in colorectal cancer. Gastroenterology 138(6): 2073-2087.

Calado RT, Young NS (2009) Telomere diseases. N Engl J Med 361(24): 2353-2365.

Cao Y, Li H, Deb S, Liu JP (2002) TERT regulates cell survival independent of telomerase enzymatic activity. Oncogene 21(20): 3130-3138.

Cunningham D, Atkin W, Lenz HJ, Lynch HT, Minsky B, Nordlinger B, Starling N (2010) Colorectal cancer. Lancet 375(9719): 1030-1047.

de Kok JB, Roelofs RW, Giesendorf BA (2005) Normalization of gene expression measurements in tumor tissues: comparison of 13 endogenous control genes. Lab Invest 85(1): 154-159.

Del Bufalo D, Rizzo A, Trisciuoglio D, Cardinali G, Torrisi MR, ZangemeisterWittke U, Zupi G, Biroccio A (2005) Involvement of hTERT in apoptosis induced by interference with Bcl-2 expression and function. Cell Death Differ 12(11): 1429-1438.

Dolcetti R, De Rossi A (2012) Telomere/telomerase interplay in virus-driven and virus-independent lymphomagenesis: Pathogenic and clinical implications. Med Res Rev 32(2): 233-253.

Engstrom PF, Arnoletti JP, Benson 3rd AB, Chen YJ, Choti MA, Cooper HS, Covey A, Dilawari RA, Early DS, Enzinger PC, Fakih MG, Fleshman Jr J, Fuchs C, Grem JL, Kiel K, Knol JA, Leong LA, Lin E, Mulcahy MF, Rao S, Ryan DP, Saltz L, Shibata D, Skibber JM, Sofocleous C, Thomas J, Venook AP, Willett C (2009) NCCN clinical practice guidelines in oncology: colon cancer. J Natl Compr Canc Netw 7(8): 778-831.

Folini M, Bandiera R, Millo E, Gandellini P, Sozzi G, Gasparini P, Longoni N, Binda M, Daidone MG, Berg K, Zaffaroni N (2007) Photochemically enhanced delivery of a cell-penetrating peptide nucleic acid conjugate targeting human telomerase reverse transcriptase: effects on telomere status and proliferative potential of human prostate cancer cells. Cell Prolif 40(6): 905-920.

Frías C, Morán A, de Juan C, Ortega P, Fernández-Marcelo T, SánchezPernaute A, Torres AJ, Díaz-Rubio E, Benito M, Iniesta P (2009) Telomere function in colorectal cancer. World J Gastrointest Oncol 1(1): 3-11.

Garcia-Aranda C, de Juan C, Diaz-Lopez A, Sanchez-Pernaute A, Torres AJ, Diaz-Rubio E, Balibrea JL, Benito M, Iniesta P (2006) Correlation of telomere lenght, telomerase activity, and telomeric-repeat binding factor 1 expression in colorectal carcinoma. Prognostic indications. Cancer 106(3): $541-551$.

Gertler R, Rosemberg R, Stricker D, Friederichs J, Hoos A, Werner M, Ulm K, Holzmann B, Nekarda H, Siewert JR (2004) Telomere length and human telomerase reverse transcriptase expression as markers for progression and prognosis of colorectal carcinoma. J Clin Oncol 22(10): 1807-1814.

Gertler R, Rosenberg R, Stricker D, Werner M, Lassmann S, Ulm K, Nekarda H, Siewert JR (2002) Prognostic potential of the telomerase subunit human telomerase reverse transcriptase in tumor tissue and nontumorous mucosa from patients with colorectal carcinoma. Cancer 95(10): 2103-2111.

Gill S, Loprinzi CL, Sargent DJ, Thomé SD, Alberts SR, Haller DG, Benedetti J, Francini G, Shepherd LE, Francois Seitz J, Labianca R, Chen W, Cha SS, Heldebrant MP, Goldberg RM (2004) Pooled analysis of fluorouracilbased adjuvant therapy for stage II and III colon cancer: who benefits and by how much? J Clin Oncol 22(10): 1797-1806.

Harrell Jr FE, Lee KL, Mark DB (1996) Multivariable prognostic models: issues in developing models, evaluating assumptions and adequacy, and measuring and reducing errors. Stat Med 15(4): 361-387.

Hastie ND, Dempster M, Dunlop MG, Thompson AM, Green DK, Allshire RC (1990) Telomere reduction in human colorectal carcinoma and with ageing. Nature 346(6287): 866-868.

Jemal A, Bray F, Center MM, Ferlay J, Ward E, Forman D (2011) Global cancer statistics. CA Cancer J Clin 61(2): 69-90.

Jin X, Beck S, Sohn YW, Kim JK, Kim SH, Yin J, Pian X, Kim SC, Choi YJ, Kim H (2010) Human telomerase catalytic subunit (hTERT) suppresses p53-mediated anti-apoptotic response via induction of basic fibroblast growth factor. Exp Mol Med 42(8): 574-582.
Kawanishi-Tabata R, Lopez F, Fratantonio S, Kim N, Goldblum J, Tubbs R, Elson P, Lavery I, Bukowski RM, Ganapathi R, Ganapathi MK (2002) Telomerase activity in stage II colorectal cancer. Cancer 95(9): $1834-1839$.

Kelland L (2007) Targeting the limitless replicative potential of cancer: the telomerase/telomere pathway. Clin Cancer Res 13(17): 4960-4963.

Kim GP, Colangelo LH, Paik S, O'Connell MJ, Kirsch IR, Allegra C, Wolmark N (2007) Predictive value of microsatellite instability-high remains controversial. J ClinOncol 25(30): 4857-4858.

Kojima K, Hiyama E, Otani K, Ohtaki M, Fukuba I, Fukuda E, Sueda T, Hiyama K (2011) Telomerase activation without shortening of telomeric $3^{\prime}$-overhang is a poor prognostic factor in human colorectal cancer. Cancer Sci 102(2): 330-335.

Maláska J, Kunická Z, Borský M, Sklenicková M, Novotná M, Fajkusová L, Zaloudík J, Fajkus J (2004) Telomerase as a diagnostic and predictive marker in colorectal carcinoma. Neoplasma 51(2): 90-96.

Markowitz SD, Bertagnolli MM (2009) Molecular origins of cancer: Molecular basis of colorectal cancer. N Engl J Med 361(25): 2449-2460.

Massard C, Zermati Y, Pauleau AL, Larochette N, Métivier D, Sabatier L, Kroemer G, Soria JC (2006) hTERT: a novel endogenous inhibitor of the mitochondrial cell death pathway. Oncogene 25(33): 4505-4514.

Menin C, Scaini MC, De Salvo GL, Biscuola M, Quaggio M, Esposito G, Belluco C, Montagna M, Agata S, D'Andrea E, Nitti D, Amadori A, Bertorelle R (2006) Association between MDM2-SNP309 and age at colorectal cancer diagnosis according to $\mathrm{p} 53$ mutation status. J Natl Cancer Inst 98(4): 285-288.

Nakamura TM, Morin GB, Chapman KB, Weinrich SL, Andrews WH, Lingner J, Harley CB, Cech TR (1997) Telomerase catalytic subunit homologs from fission yeast and human. Science 277(5328): 955-959.

Nowak J, Januszkiewicz D, Lewandowski K, Nowicka-Kujawska K, Pernak M, Rembowska J, Nowak T, Wysocki J (2003) Activity and expression of human telomerase in normal and malignant cells in gastric and colon cancer patients. Eur J Gastroenterol Hepatol 15(1): 75-80.

O'Connor ES, Greenblatt DY, LoConte NK, Gangnon RE, Liou JI, Heise CP, Smith MA (2011) Adjuvant chemotherapy for stage II colon cancer with poor prognostic features. J Clin Oncol 29(25): 3381-3388.

Pino MS, Chung DC (2010) The chromosomal instability pathway in colon cancer. Gastroenterology 138(6): 2059-2072.

Pucciarelli S, Agostini M, Viel A, Bertorelle R, Russo V, Toppan P, Lise M (2003) Early-age-at-onset colorectal cancer and microsatellite instability as markers of hereditary nonpolyposis colorectal cancer. Dis Colon Rectum 46(3): 305-312.

Quasar Collaborative GroupGray R, Barnwell J, McConkey C, Hills RK, Williams NS, Kerr DJ (2007) Adjuvant chemotherapy versus observation in patients with colorectal cancer: a randomised study. Lancet 370(9604): 2020-2029.

Rahman R, Latonen L, Wiman KG (2005) hTERT antagonizes p53-induced apoptosis independently of telomerase activity. Oncogene $\mathbf{2 4}(8)$ : $1320-1327$.

Rampazzo E, Bertorelle R, Serra L, Terrin L, Candiotto C, Pucciarelli S, Del Bianco P, Nitti D, De Rossi A (2010) Relationship between telomere shortening, genetic instability, and site of tumor origin in colorectal cancer. Br J Cancer 102(8): 1300-1305.

Ribic CM, Sargent DJ, Moore MJ, Thibodeau SN, French AJ, Goldberg RM, Hamilton SR, Laurent-Puig P, Gryfe R, Shepherd LE, Tu D, Redston M, Gallinger S (2003) Tumor microsatellite-instability status as a predictor of benefit from fluorouracil-based adjuvant chemotherapy for colon cancer. N Engl J Med 349(3): 247-257.

Rufer N, Migliaccio M, Antonchuk J, Humphries RK, Roosnek E, Lansdorp PM (2001) Transfer of the human telomerase reverse transcriptase (TERT) gene into T lymphocytes results in extension of replicative potential. Blood 98(3): 597-603.

Safont MJ, Gil M, Sirera R, Jantus-Lewintre E, Sanmartín E, Gallach S, Caballero C, Del Pozo N, Palomares E, Camps C (2011) The prognostic value of hTERT expression levels in advanced-stage colorectal cancer patients: a comparison between tissue and serum expression. Clin Transl Oncol 13(6): 396-400.

Saleh S, Lam AK, HO YH (2008) Real-time PCR quantification of human telomerase reverse transcriptase (hTERT) in colorectal cancer. Pathology 40(1): 25-30.

Sanz-Casla MT, Vidaurreta M, Sanchez-Rueda D, Maestro ML, Arroyo M, Cerdan FJ (2005) Telomerase activity as prognostic factor in colorectal cancer. Onkologie 28(11): 553-557. 
Sargent DJ, Marsoni S, Monges G, Thibodeau SN, Labianca R, Hamilton SR, French AJ, Kabat B, Foster NR, Torri V, Ribic C, Grothey A,

Moore M, Zaniboni A, Seitz JF, Sinicrope F, Gallinger S (2010)

Defective mismatch repair as a predictive marker for lack of efficacy of fluorouracil-based adjuvant therapy in colon cancer. J Clin Oncol 28(20): 3219-3226.

Takagi S, Kinouchi Y, Hiwatashi N, Chida M, Nagashima F, Takahashi S, Negoro K, Shimosegawa T, Toyota T (1999) Telomere shortening and the clinicopathologic characteristics of human colorectal carcinomas. Cancer 86(8): 1431-1436.

Tatsumoto N, Hiyama E, Murakami Y, Imamura Y, Shay JW, Matsuura Y, Yokoyama T (2000) High telomerase activity is an independent prognostic indicator of poor outcome in colorectal cancer. Clin Cancer Res 6(7): 2696-2701.

Terrin L, Dolcetti R, Corradini I, Indraccolo S, Dal Col J, Bertorelle R, Bonaldi L, Esposito G, De Rossi A (2007) hTERT inhibits the Epstein-Barr virus lytic cycle and promotes the proliferation of primary B lymphocytes: implications for EBV-driven lymphomagenesis. Int J Cancer 121(3): 576-578.

Terrin L, Rampazzo E, Pucciarelli S, Agostini M, Bertorelle R, Esposito G, DelBianco P, Nitti D, De Rossi A (2008) Relationship between tumor and plasma levels of hTERT mRNA in patients with colorectal cancer: implications for monitoring of neoplastic disease. Clin Cancer Res 14(22): 7444-7451.

Therneau M, Grambsch TM (2000) Modelling survival data. Springer: New York.

Van Cutsem E, Köhne CH, Hitre E, Zaluski J, Chang Chien CR, Makhson A, D’Haens G, Pintér T, Lim R, Bodoky G, Roh JK, Folprecht G, Ruff P, Stroh C, Tejpar S, Schlichting M, Nippgen J, Rougier P (2009) Cetuximab and chemotherapy as initial treatment for metastatic colorectal cancer. $N$ Engl $J$ Med 360(14): 1408-1417.

Vidaurreta M, Maestro ML, Rafael S, Veganzones S, Sanz-Casla MT, Cerdán J, Arroyo M (2007) Telomerase activity in colorectal cancer, prognostic factor and implications in the microsatellite instability pathway. Word $J$ Gastroenterol 13(28): 3868-3872.

This work is published under the standard license to publish agreement. After 12 months the work will become freely available and the license terms will switch to a Creative Commons AttributionNonCommercial-Share Alike 3.0 Unported License. 